doi:10.4149/neo_2015_075

\title{
Prognostic value of interim and restaging PET/CT in Hodgkin lymphoma. Results of the CHEAP (Chemotherapy Effectiveness Assessment by PET/CT) study - long term observation
}

\author{
Z. MILTENYI ${ }^{1, *}$, S. BARNA ${ }^{2}$, I. GARAI ${ }^{2}$, Z. SIMON ${ }^{1}$, A. JONA ${ }^{1}$, F. MAGYARI ${ }^{1}$, M. GERGELY ${ }^{3}$, Z. NAGY ${ }^{4}$, K. KERESZTES $^{5}$, P. PETTENDI $^{6}$, A. ILLES ${ }^{1}$
}

${ }^{1}$ Internal Medicine, University of Debrecen, Debrecen, Hungary; ${ }^{2}$ Scanomed Ltd, Debrecen, Hungary; ${ }^{3}$ University of Debrecen, Debrecen, Hungary; ${ }^{4}$ MISEK Ltd, Miskolc, Hungary; ${ }^{5}$ Andras Josa Hospital, Nyiregyhaza, Hungary; ${ }^{6}$ Geza Hetenyi Hospital, Szolnok, Hungary

*Correspondence: mil03@freemail.hu

Received September 1, 2014 / Accepted February 11, 2015

\begin{abstract}
Very few studies have determined the prognostic value of interim and restaging PET/CT in patients with Hodgkin lymphoma using current standard of care therapy outside clinical trials. We analyzed the effect of the results of interim and restaging PET/CT on the survival (overall- and relapse-free) in patients who received standard first-line treatment based on the stage of disease and risk factors. We investigated the differences between the relapse and non-relapse groups based on the clinical pathological characteristics of patients who had positive interim PET/CT results.

Between January 1, 2007 and December 31, 2011, the staging, interim and restaging PET/CT scans of patients with Hodgkin lymphoma were analyzed. The Deauville criteria were used for the evaluation of interim PET/CT scans.

One hundred and thirteen Hodgkin lymphoma patients underwent staging, interim and restaging PET/CT scans. None of the therapy was modified based on the interim PET/CT results. The median follow-up time was 43.5 months. A total of 62 early stage patients and 51 advanced stage patients were identified. The five-year overall survival rates were $93.4 \%$ in the interim PET negative group and $58 \%$ in the interim PET positive group $(\mathrm{p}<0.001)$. The five-year relapse-free survival rates for the negative and positive groups were $92.7 \%$ and $40.8 \%$, respectively $(\mathrm{p}<0.001)$. The negative predictive value was $100 \%$ in the early stage group and $82.35 \%$ in the advanced stage group. By comparison, the positive predictive values were $53.8 \%$ and $58.8 \%$, respectively, in these two groups. In the interim PET positive group, patients over 40 years of age had a significantly higher probability of relapse $(\mathrm{p}=0.057)$.

The routine clinical use of interim PET/CT is highly recommended based on our investigation. However, patients with positive interim $\mathrm{PET} / \mathrm{CT}$ results required frequent additional evaluations.
\end{abstract}

Key words: predictive value, Hodgkin lymphoma, PET/CT

Combined computed tomography and positron emission tomography using 2-deoxy-2-[fluorine-18]fluoro-D-glucose $\left({ }^{18} \mathrm{FDG}-\mathrm{PET} / \mathrm{CT}\right)$ has recently become a valuable modality in the treatment of lymphoma patients because it provides exact staging and objective determination of early therapeutic response. Interim ${ }^{18} \mathrm{FDG}$-PET/CT offers an excellent negative predictive value for Hodgkin lymphoma (HL); however, the role of positive interim PET is less clear because its positive predictive value is not sufficiently high $[1,2]$. Research by Gallamini et al. has shown that the prognostic value of ${ }^{18} \mathrm{FDG}$-PET performed after the second cycle of adriamycin-bleomycin-vinblastine-dacarbazine (ABVD) treatment outperforms the prognostic value of the inter- national prognostic score [3]. Therefore, interim PET can provide valuable help in avoiding under- and overtreatment because response-based therapy can be used as opposed to risk-adapted treatment, or these two could be combined [3]. Recently, many ongoing clinical studies of early and advanced stage patients have evaluated whether modification of treatment based on the results of interim PET/CT scan could have improved patient outcomes. In addition, the exclusion of radiotherapy in interim PET negative early stage patients and the necessity of therapy intensification in interim PET positive advanced stage patients were investigated [4]. However, very few studies have assessed the accurate prognostic value of interim and restaging PET/ 
CT on long-term patient using standard therapy (based on current treatment guidelines).

Furthermore, the role of interim PET/CT among patients who were treated outside clinical trials has not been accurately determined. We analyzed the effect of the results of interim and restaging PET/CT on the survival (overall- and relapse-free) in patients who received standard first-line treatment based on the stage of disease and risk factors. We investigated the differences between the relapse and non-relapse groups based on the clinical pathological characteristics of patients who had positive interim $\mathrm{PET} / \mathrm{CT}$ results.

\section{Patients and methods}

We investigated the role of interim and restaging PET/ CT scans in HL patients as part of the Chemotherapy Effectiveness Assessment by PET/CT (CHEAP) observational study between January 1, 2007 and December 31, 2011 at the Institute of Internal Medicine, University of Debrecen. The primary chemotherapy consisted of ABVD during a 28 day cycle. ${ }^{18} \mathrm{FDG}-\mathrm{PET} / \mathrm{CT}$ scans were performed according to a standard clinical protocol using the same Philips Timeof-Flight (TOF) PET/CT system. Two experienced nuclear medicine physicians read the scan images independently. Early stage patients received involved field radiotherapy (30-36 Gy) after chemotherapy. Based on the European Organization for

Table 1. Deauville criteria

\begin{tabular}{|c|c|}
\hline 1. No uptake. & PET negative \\
\hline 2. Uptake $\leq$ mediastinum & \\
\hline 3. Uptake $>$ mediastinum $\leq$ liver & \\
\hline 4. Uptake moderately increased above liver at any sites & PET positive \\
\hline $\begin{array}{l}\text { 5. Markedly increased uptake above liver at any site } \\
\text { including new sites }\end{array}$ & \\
\hline
\end{tabular}

Table 2. Clinical features of patients

\begin{tabular}{lc}
\hline \multicolumn{2}{c}{ Patients } \\
\hline Men/women & $57 / 56$ \\
\hline mean age & 37.94 years (17-79 years) \\
\hline$<$ 40 years old & 76 \\
\hline Histological subtypes & \\
$\quad$ NLP & 4 \\
MC & 30 \\
NS & 50 \\
LR & 22 \\
LD & 1 \\
ND & 5 \\
\hline Stage (I, II) & 62 \\
\hline Stage (III, IV) & 51 \\
\hline B symptom & 59 \\
\hline NLP: nodular lymphocyte predominant, MC: mixed cellularity, NS: \\
nodular sclerosis, LR: lymphocyte rich, LD: lymphocyte depleted ND: not \\
differentiated
\end{tabular}

Research and Treatment (EORTC) prognostic score, patients with a favorable prognosis received 2-4 cycles of chemotherapy whereas patients with an unfavorable prognosis received 4-6 cycles. Advanced stage disease patients received 6-8 cycles of ABVD treatment extended with involved field radiotherapy (30-36 Gy) in the case of residual disease or bulky tumor detected at staging. After the second cycle of chemotherapy, interim ${ }^{18} \mathrm{FDG}$-PET/CT scan was performed for each patient preferably a few days (2-3 days) before the third cycle of chemotherapy. Restaging scans were performed 6 weeks after treatment in the case of chemotherapy alone, and 8 weeks after combined (chemotherapy and radiotherapy) modality treatment. Six hours of fasting was mandatory prior to the ${ }^{18} \mathrm{FDG}$ PET/CT scan. The scan was not performed in patients with a blood glucose level above $11 \mathrm{mmol} / \mathrm{l}$. The interim PET/ CT results were evaluated visually (in a qualitative manner) based on the Deauville criteria (Table 1). Because these criteria were published in 2010, scans performed before that time were reevaluated. In addition, the maximum value in the region of interest (ROI) placed on the standardized uptake value (SUV) image was also used as a semi-quantitative measure in the evaluation. The SUVmax value of lesions having active metabolism were compared to the liver SUVmax value, and all these provided accurate rankings [5]. None of the therapy was modified based upon interim PET/CT results alone; only in those few cases in which there was clinical evidence of disease progression was their therapy modified. We investigated the occurrence of relapses, and the differences between the relapse and non-relapse groups based on the clinical pathological characteristics of the disease (biopsy, stage, age, lactate dehydrogenase (LDH) level at the time of initial diagnosis, EORTC ranking according to the early stage and international prognostic score (IPS) in advanced stage) for patients who had positive interim PET/CT results. Statistical analysis was performed using SPSS-15 software. Survival data were calculated by Kaplan-Meier analysis and the results were compared with the log-rank test. Approval by the Institutional Review Board (IRB) or an ethic committee was not been required because this was an observational, retrospective study, therapy change was not based on the PET/CT results, and the patients were treated according to the current standard of care.

\section{Results}

One hundred and thirteen HL patients (57 male and 56 female) underwent staging, interim and restaging PET/CT scans. The clinical parameters of patients are summarized in Table 2 . The median age of patients was 34 (17-79) years at the time of diagnosis and the median average follow up time was 43.5 (7-70) months. Sixty-two patients had early stage disease, 51 had advanced stage disease and 59 patients had B symptoms. Interim PET/CT results were negative (intPET-) in 83 cases $(73.45 \%)$, and positive (intPET+) for 30 patients (26.55\%). The negative predictive value (NPV) was $92.7 \%$ and the positive predictive value (PPV) was $56.6 \%$. The five- 

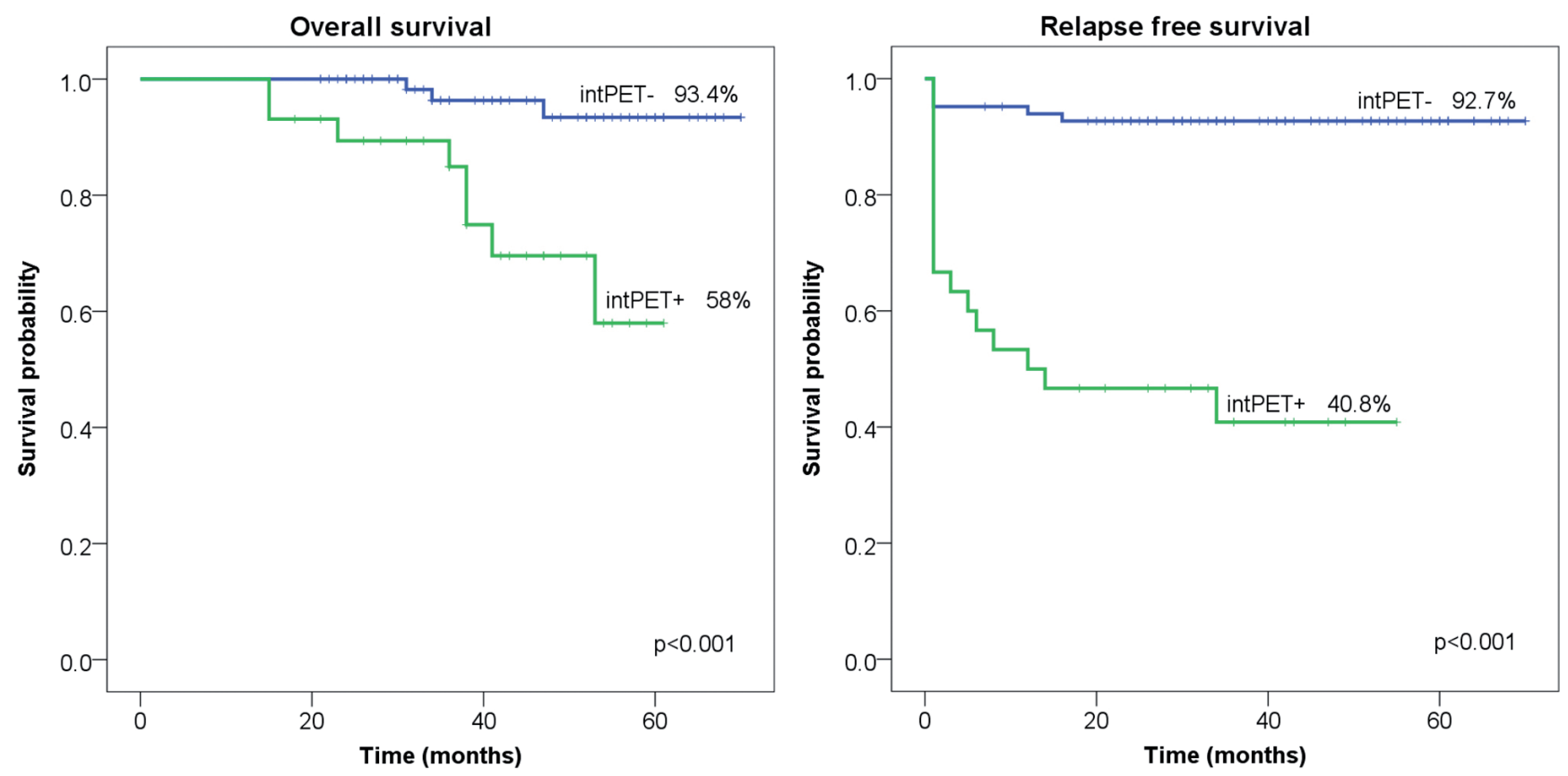

Figure 1. Overall- and relapse free survival (OS and RFS) in interim PET positive and negative groups (113 patients).

year overall survival (OS) was $93.4 \%$ in the intPET- group in contrast to only $58 \%$ in the intPET + group $(\mathrm{p}<0.001)$. The five-year relapse-free survival (RFS) was $92.7 \%$ and $40.8 \%$ $(\mathrm{p}<0.001)$ for the intPET- and intPET + groups, respectively (Figure 1).

Early stage. In early stage patients, NPV was $100 \%$ whereas PPV was $53.8 \%$. Both the 5 year overall survival (intPET- 94.3\% vs. intPET $+40.4 \%, \mathrm{p}=0.017$ ) and relapse-free survival (RFS) (intPET- 100\% vs. intPET+35.9\%, p<0.0001) significantly differed based on interim PET scan (Figure 2). Data of the favorable and unfavorable prognostic groups were evaluated separately. NPV was $100 \%$ for both groups, while PPV 25\% and 66.67\%, respectively. No relapse was noted in the intPET-group during long term follow-up. Two deaths occurred in this group, both caused by a solid neoplasm (gynecological tumor and carcinoma planocellulare). In the intPET+ group (13 patients), 7 relapses or progressions were observed; from these, 5 patients had a positive restaging PET/CT scan. Two patients had positive interim PET scans and clinical signs of progression; as a result, treatment was changed in these cases. For those 6 patients who had positive interim PET/CT results, but negative restaging PET/CT, no relapse occurred during the follow-up period. In all cases in which restaging had positive results, we observed refractory disease or relapse. All relapse patients were early stage, unfavorable patients, except for one case. Only one patient relapsed after one year (34 months). Three patients died due to HL in the intPET+ early stage group.

Advanced stage. In advanced stage patients, NPV was $82.35 \%$ whereas PPV was $58.8 \%$. Both the 5 -year OS (intPET-
92.3\% vs intPET $+65.6 \%, \mathrm{p}=0.024$ ), and RFS (intPET- $81.9 \%$ vs. intPET $+41.2 \%, \mathrm{p}=0.002$ ) were significantly different in this group also, based on interim PET/CT results (Figure 3). In patients with IPS $0-3, \mathrm{NPV}$ and PPV was $94.44 \%$ and $45.45 \%$, respectively, while in patients with IPS $\geq 4$, NPV was $66.67 \%$ and PPV was $100 \%$. In the interim PET negative group

Table 3. Clinical features of interim PET positive patients

\begin{tabular}{lccc}
\hline & relapsed & non relapsed & $\mathbf{p}$ \\
\hline Patients & 17 & 13 & \\
\hline Men/women & $8 / 9$ & $9 / 4$ & NS \\
\hline mean age (years) & 47,3 & 35,9 & NS \\
\hline$<$ 40 years old & $\mathbf{8}$ & $\mathbf{1 1}$ & $\mathbf{p}=\mathbf{0 . 0 5 7}$ \\
\hline Histological subtypes & & & NS \\
$\quad$ MC & $\mathbf{9}$ & 3 & \\
$\quad$ NS & 5 & 4 & \\
LR & 2 & 5 & \\
ND & 1 & 1 & NS \\
\hline Early stage & 7 & 6 & \\
$\quad$ favourable & 1 & 3 & \\
$\quad$ unfavoruable & 6 & 3 & NS \\
Advanced stage & 10 & 7 & NS \\
\hline IPS: 0-3 & 5 & 5 & NS \\
IPS $\geq \mathbf{4}$ & 3 & 0 & 7 \\
\hline B sign & 14 & $\mathbf{2} 1 \mathbf{1 1}$ \\
\hline LDH (abnormal/normál) & $\mathbf{5 / 1 2}$ & & \\
\hline
\end{tabular}

NS: not significant, LDH normal range.: 135-220U/1, NLP: nodular lymphocyte predominant, MC: mixed cellularity, NS: nodular sclerosis, LR: lymphocyte rich, LD: lymphocyte depleted ND: not differentiated 

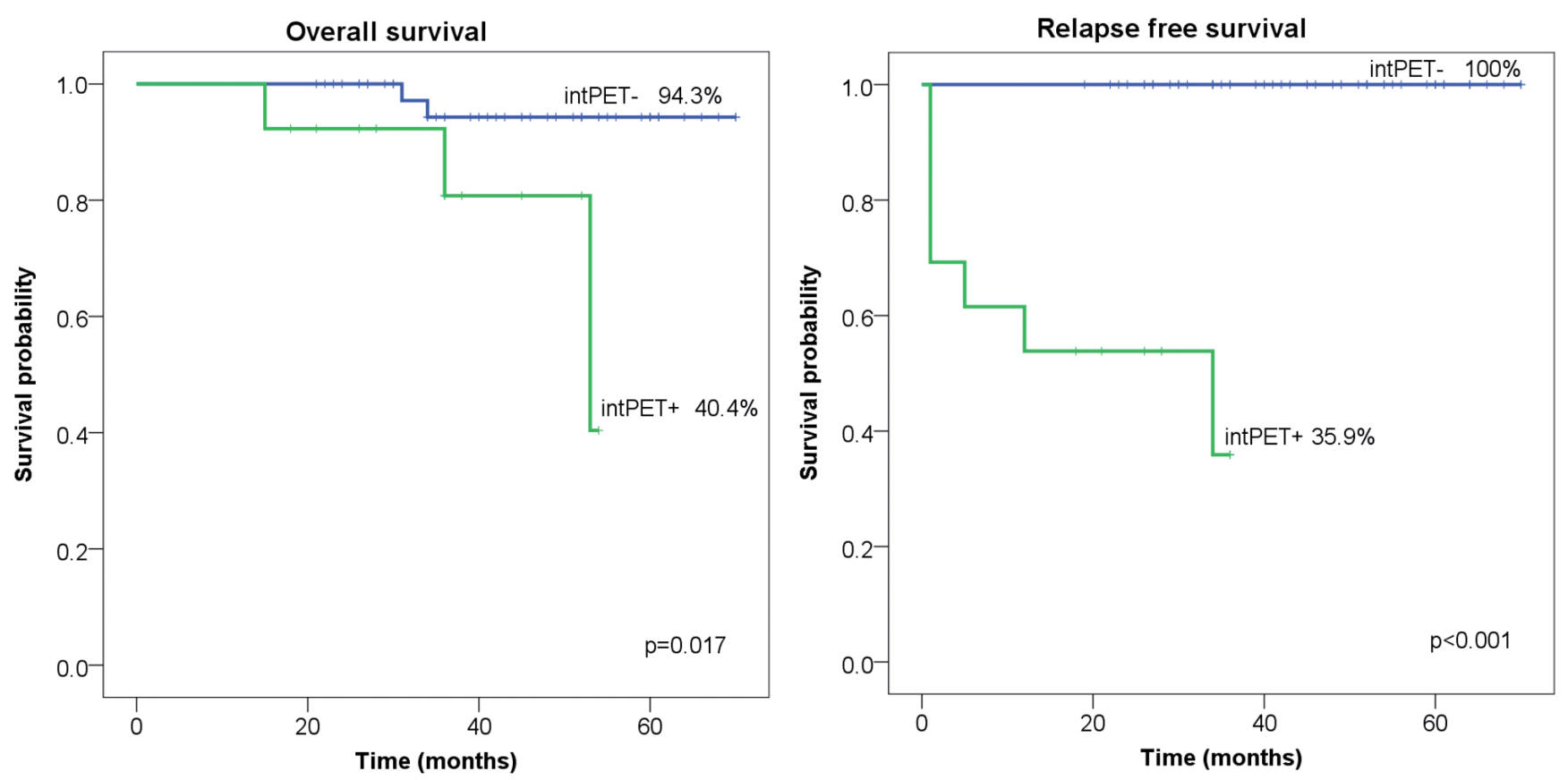

Figure 2. Overall- and relapse free survival (OS and RFS) in early stage Hodgkin-lymphoma group (62 patients).
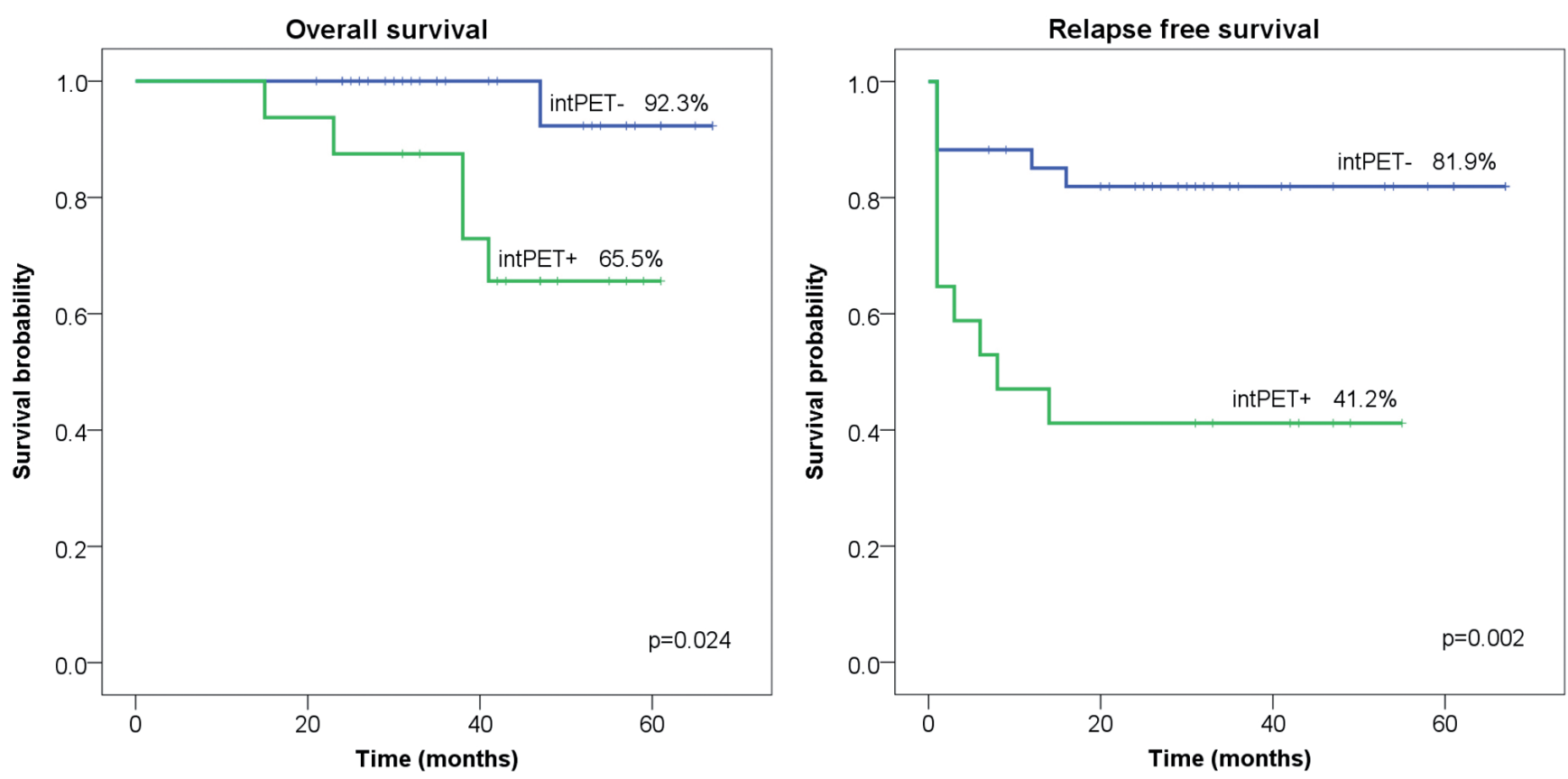

Figure 3. Overall- and relapse free survival (OS and RFS) in advanced stage Hodgkin-lymphoma group (51 patients).

(34 patients), we observed 6 relapses of young patients (average age: 25.2 years, range: 22-30 years). Relapsing patients were chemosensitive; all of them underwent successful autologous transplantation, and no relapse or death occurred during the follow-up period (average: 51 months, range: 24-65 months). Only one non-relapse patient died due to small cell lung cancer. False negative cases were only found in advanced stage patients based on interim PET/CT results. In the intPET+ 
group, 10 patients relapsed, 7 patients were false positive (all of them had negative restaging PET/CT). No relapses occurred during the follow-up period or the average follow-up period (31-55 months and 42.85 months, respectively). Five patients died in this group, all of them due to HL. Similarly to the early stage group, we observed refractory disease or relapse at 1 year in patients with positive restaging scans. Only two relapses occurred after 1 year (14 and 16 months later).

Deaths. Eleven deaths occurred in the entire group of 113 patients. Three patients died due to secondary tumor in the intPET- group. Eight patients died due to HL in the intPET+ group, of whom 4 patients were over 70 years of age, 5 patients had refractory disease, and 3 patients underwent autologous hematopoietic stem cells transplantation (AHSCT).

Relapses. In the intPET+ group, mainly early relapse (within 12 months) and refractory disease was observed. Only one patient relapsed after more than one year (34 months after first-line treatment).

In the intPET+ group (total of 30 patients), the differences between the relapse and non-relapse patients were compared (Figure 3 ). From the clinical features, only age over 40 (Fischer exact test $\mathrm{p}=0.057$ ) was a risk factor of relapse. There was no significant difference between the two groups based on sex, average age, B symptoms, international prognostic score (IPS), serum albumin level, hemoglobin level, lymphocyte number, and sedimentation rate.

\section{Discussion}

It is an old clinical observation that a quick therapeutic response (regression in the peripheral regions after the first or second chemotherapy cycle) indicates better survival and remission rates. Gallamini et al. showed that the prognostic value of ${ }^{18} \mathrm{FDG}$-PET after the second cycle of ABVD treatment was higher than that for IPS, and that interim PET scan can provide valuable help in avoiding under- or over-treatments [3]. In that study, 260 patients with HL underwent interim $\mathrm{PET} / \mathrm{CT}$ scans after the second cycle of ABVD treatment without any therapy changes thereafter. NPV was $92 \%$ and PPV 93\% for the interim PET/CT [3]. However, that study also included early and advanced stage patients (from stage IIA with unfavorable prognostic values to stage IV) and not every early stage patients received radiotherapy (only $40 \%$ for stage IIA patients with unfavorable prognosis and $66 \%$ of patients in stage IIB).

According to the International Validation Study (IVS), 261 advanced stage HL patients (stage IIA with unfavorable prognosis and stage IIB to IVB) were diagnosed internationally from 8 countries. Interim PET/CT was performed after two cycles of ABVD, and no therapy modification was based on these results. The average follow-up time was 38 months. The three-year progression-free survival was $28 \%$ and $95 \%$ for PET positive and PET negative patients (NPV: 95\%, PPV: $64.2 \%$ ) [1]. In our cohort of patients, results similar to IVS were obtained with almost one year longer follow up time (Table 4). Although our PPV value was lower than that of IVS in 2013, it did not differ significantly for the early and advanced stage groups. However, if the early stage group was analyzed based on prognostic values, then PPV was higher for the group with an unfavorable prognosis. Therefore, we assumed that in the case of a positive interim PET/CT result, conventional risk factors should also be taken into account for determining a possible risk of relapse; hence, a risk- and response-based treatment could be applied. Very few studies have examined the prognostic value of interim PET/CT with conventional prognostic factors for early stage HL patients. Unfortunately, results from these studies were not comparable either, because neither the patient groups, nor the treatment methods were similar, and the time of interim PET/CT along with the evaluation was also different [6-11] (Table 5). Entire groups of patients with an unfavorable prognosis in the early stage, or only those patients who had bulky tumor are generally treated like an advanced stage group. Therefore, an accurate determination of under- and overtreatment was not possible. Hutchings et al. used interim PET/CT after 2-3 cycles of ABVD in 57 early stage patients, and evaluated their results according to three categories (negative, minimal residual uptake and positive). Their results showed a PPV of $30 \%$ and NPV of $95 \%$; 2 -year PFS for intPET- patients was $97 \%$ and for intPET+ was $70 \%$. While in the early stage group, only two out of seven

Table 4. Comparsion of results of Gallamini-, International Validation- and CHEAP study

\begin{tabular}{lcccc}
\hline Study & $\begin{array}{c}\text { JCO 2007 (3) } \\
\text { median follow-up 2.19 year }\end{array}$ & $\begin{array}{c}\text { IVS 2011 (1) } \\
\text { median follow-up 3.13 year }\end{array}$ & $\begin{array}{c}\text { IVS 2013 (14) } \\
\text { median follow-up 3.08 year }\end{array}$ & $\begin{array}{c}\text { CHEAP } \\
\text { median follow-up 4.04 year }\end{array}$ \\
\hline Evaluation criteria & Gallamini criteria & Deauville criteria & Deauville criteria & Deauville criteria \\
True positive & 44 & 30 & 33 & 17 \\
True negative & 194 & 204 & 203 & 77 \\
False positive & 6 & 16 & 12 & 13 \\
False negative & 11 & 11 & 12 & 6 \\
Sensitivity & 0.81 & 0.732 & 0.73 & 0.739 \\
Specificity & 0.97 & 0.927 & 0.94 & 0.855 \\
PPV & 0.93 & 0.652 & 0.73 & 0.566 \\
NPV & 0.92 & 0.949 & 0.94 & 0.927 \\
\hline
\end{tabular}


Table 5. PPV and NPV of interim PET/CT in early stage Hodgkin lymphoma patients

\begin{tabular}{|c|c|c|c|c|c|}
\hline & Hutchings (6) & Zinzani (7) & Straus $(8)$ & Cerci (9) & CHEAP \\
\hline Evaluation criteria & Hutchings criteria & Gallamini criteria & IHP criteria & Gallamini criteria & Deauville criteria \\
\hline PPV & $30 \%$ & $52.63 \%{ }^{\times}$ & $50 \%$ & $44 \%$ & $53.8 \%$ \\
\hline NPV & $95 \%$ & $95.31 \%$ & $86 \%$ & $94 \%$ & $100 \%$ \\
\hline
\end{tabular}

*estimated value based on the results of the publication

intPET + patients relapsed, in the advanced stage group, all six intPET+ patients relapsed in two years [6].

Zinzani et al. performed interim PET/CT scans in 147 early stage HL patients (stage I-IIA) after 2 cycles of ABVD, but their treatment was not uniform, as they applied 6 cycles of ABVD (57 patients) or 4 cycles of ABVD with involved-field radiotherapy (IFRT) (90 patients). It is unknown if there was any difference between the two groups with respect to the later relapses [7].

Strauss et al. analyzed the prognostic value of interim PET of 88 patients in stage I-II disease with non-bulky tumors using chemotherapy (doxorubicin, vinblastine and gemcitabine) other than standard ABVD treatment. Two-year PFS was $88 \%$ and $54 \%(\mathrm{p}=0.0009)$ for the intPET- and intPET+ groups, respectively. PPV was 50\% while NPV was $86 \%$ [8].

Cerci et al. studied 43 patients in early stage. These patients received 4-6 cycles of ABVD in the early stage, stage III patients 6-8 cycles, and stage IV patients 8 cycles. If an early stage patient with good prognosis and bulky tumor received only 4 cycles of chemotherapy, they were administered IFRT as well. PPV was 44\% and NPV 94\%. The three-year event-free survival (EFS) and OS were found to be $90 \%$ and $50 \%$, respectively, in the intPET- and intPET+ groups $(\mathrm{p}=0.002)$ [9].

Barnes et al. conducted a retrospective study of early stage patients with non-bulky tumor who received ABVD treatment and, in 56\% of the cases, IFRT as well. Restaging PET/ $\mathrm{CT}$ (rPET) values were found to be significant in terms of the survival rates. PFS was $94 \%$ and $54 \%$ for rPET- and rPET+ $(\mathrm{p}<0.0001)$ patients, respectively, while for interim PET, PFS was $87 \%$ and $91 \%(p=0.57)$. Interim PET was used after $2-4$ cycles of chemotherapy and individual evaluations were performed [10].

Filippi et al. treated 80 early stage (I-IIA) HL patients with 3-4 cycles of ABVD plus 30 Gy IFRT. Interim PET showed negative results for $87.5 \%$ of the patients; among them, only one relapse was identified. Interim PET had positive results for $12.5 \%$ and there was no relapse among these patients. The three-year OS and progression-free survival (PFS) was found to be $97 \%$ and $98.4 \%$, respectively, for the intPET-group, while these were $100 \%$ and $100 \%$ for the intPET+ group $(p=0.63)$. Overall, no treatment modification was suggested based on the outcome of interim PET [11].

Zinzani et al. treated 157 advanced stage patients with ABVD (stage IIB-IVB). Interim PET was performed after 2 cycles, and 34 patients were found to be intPET+ and 123 were intPET-. Fourteen patients with relapse or progressive disease were in the intPET-group and 17 patients in the intPET+ group [7].

Cerci et al. evaluated 61 advanced stage patients and used $6-8$ cycles of ABVD for patients in stage III, and 8 cycles in stage IV. Out of 40 patients, 4 relapsed in the intPET-group, while in the intPET + group, 12 relapsed out of 21 patients. The three-year EFS was 94\% and 55\% ( $<<0.001)$, and the OS was $90 \%$ and $50 \%(\mathrm{p}=0.002)$ for the intPET- and intPET+ groups, respectively. PPV was 57\% while NPV was 90\% [9].

Different studies have indicated that unified conclusions cannot be reached for everyday clinical routine care because of the various patient groups, interim PET/CT evaluation and treatment methods. Similarly, recent prospective studies with both early and advanced stages are not unified in terms of patient groups and treatment of choice [4]. PPV values were found to improve in patient with advanced stages compared with early stages as indicated on Table 6 . To the best of our understanding, interim PET/CT has a role as a prognostic indicator; however, its role in guiding safe therapeutic changes remains unclear at this time [12].

In international studies related to our research on interim PET/CT positive patients, both total- and progression-free survival was worse. However, because low PPV for the selection of patients for changes in therapy based on interim PET/CT scan is important, their probabilities of survival are improved. However, no change in therapy will be made for those (false positive cases) who would receive over treatment, leading to

Table 6. PPV and NPVof interim PET/CT in advanced stage Hodgkin lymphoma patients

\begin{tabular}{|c|c|c|c|c|c|c|}
\hline & JCO 2007 (3) & IVS 2011 (1) & IVS 2013 (14) & Zinzani (7) & Cerci (9) & CHEAP \\
\hline Evaluation criteria & Gallamini criteria & Deauville criteria & Deauville criteria & Gallamini criteria & Gallamini criteria & Deauville criteria \\
\hline PPV & $93 \%$ & $65.2 \%$ & $73 \%$ & $61.76 \%^{x}$ & $57 \%$ & $58.8 \%$ \\
\hline NPV & $92 \%$ & $94.9 \%$ & $94 \%$ & $88.61 \%{ }^{*}$ & $90 \%$ & $82.37 \%$ \\
\hline
\end{tabular}

* estimated value based on the results of the publication 
high risks of late side effects. Therefore, the question remains: How can the positive predictive value of the interim PET/CT be improved?

Le Roux et al. in 2011 compared the prognostic values of interim PET in a group of 90 patients. Patients received 4 cycles of ABVD. The therapeutic strategy was based on the prognostic factors, $\mathrm{CT}$ and interim PET/CT results. The $\mathrm{PET} / \mathrm{CT}$ images were evaluated visually in a prospective manner. The absence of residual FDG uptake compared to background was considered to be negative; all other cases were considered to have positive interim PET. The NPV was high (95\%) similarly to previous studies; however, the PPV was low (16\%). Scans were evaluated retrospectively based on the IHP criteria, Gallamini criteria and 5-point scale (London criteria). Based on the IHP criteria, the PPV was higher than $16 \%$ (25\%); however, using the Gallamini criteria, PPV was $45 \%$. The best results were found to be $55 \%$ based on the London criteria. Moreover, the PET positive predictive value improved while the NPV remained almost the same [2].

A retrospective study by Dann et al. focused on improving the PPV of interim PET, in which the so-called dynamic visual evaluation method was used to foster the positive predictive value. Although those cases in which the FDG level declined were counted to be positive, the number of lesions did not change as well as when neither the FDG uptake nor the lesion number changed. During the reevaluation of PET scans, the number of PET positive cases dropped from 24 to 6; as a result, the specificity increased to $94 \%$ (compared to $78-86 \%$ previously when the static visual inspection method was used). The five-year total- and progression-free survival in the negative group was $97 \%$ and $92 \%$, whereas in the PET positive group, corresponding values were $67 \%$ and $50 \%$, respectively. However, because two-thirds of the patients received BEACOPP (bleomycin, etoposide, adriamycin, cyclophosphamide, vincristine, procarbazine, prednisone) therapy, those results cannot be compared to studies in which ABVD was used as frontline treatment [13].

In this present study, we also evaluated classic clinical risk factors, while no such international study has been published yet. Based on our results, it is important to say that special consideration is needed for patients over the age of 40 because this had a significant effect $(\mathrm{p}=0.057)$ on relapses in our study. The $\mathrm{PPV}$ of interim PET/CT scan improved if stage and conventional prognostic values were taken into account. In the early stage group with unfavorable prognosis and the advanced stage higher risk (IPS $\geq 4$ ) group, higher PPV values were found, concluding that for therapy decisions, unified evaluation of the prognostic factors and interim PET/CT results is important. Based on this evaluation (and the over risk- and response adapted treatment), a personalized treatment could be performed.

\section{Conclusion}

Although interim PET/CT used for the early evaluation of the therapeutic response has an evident prognostic role, its contribution to therapy modification remains in question. The results of interim scans are influenced by the time of scan, the evaluation method and chemotherapy of choice. Using classic risk- and response-adapted therapy with current treatment methods, under and over treatment of patients could be avoided. Changes in the levels of biomarkers (e.g., Thymus and Activation Regulated Chemokine, soluble CD163, etc.) at the time of staging and during treatment can also help.

Based on the results of our study, it could be concluded that interim PET/CT has evident prognostic role for the evaluation of progression free- and overall survival even with long-term follow-up. Typically, a positive PET/CT results alone do not provide sufficient information on which to base a decision to modify therapy; however, taking into account, the patients' age and conventional prognostic systems with the results of PET/ CT could help identify patients with higher risks for relapse, and consequently, determine personalized treatment. Classic prognostic scores and staging by PET/CT are already known at the time of diagnosis, and there is no need for new and expensive biomarkers. All these contribute to the decision of therapy. Further improvement of the interim PET/CT evaluation is also necessary. Because the vast majority of relapses occur within one year, the use of routine PET/CT scan during long-term follow up is not recommended.

Modification of therapy based on the results of interim PET/ CT may be only possible in clinical trials today. However, for more correct results, a larger, randomized and unified international clinical trial would be necessary with a larger cohort of patients and a longer follow-up time.

\section{References}

[1] GALLAMINI A. International Validation Study of the Prognostic Role of Interim-PET Scan in ABVD-treated, Advanced Stage Hodgkin Lymphoma. 3rd International Workshop on Interim PET in Lymphoma, Menton (France), September 26-27, 2011. Available at: http://eitti.free.fr. Accessed April 2012.

[2] LE ROUX PY, GASTINNE T, LE GOUILL S, NOWAK E, BODET-MILIN C et al: Prognostic value of interim FDG $\mathrm{PET} / \mathrm{CT}$ in Hodgkin's lymphoma patients treated of interim response-adapted strategy: comparison of International Harmonization Project (IHP), Gallamini and London criteria. Eur J Nucl Med Mol Imaging.2011; 38: 1064-1071. http://dx.doi. org/10.1007/s00259-011-1741-0

[3] GALLAMINI A, HUTCHINGS M, RIGACCI L, SPECHT L, MERLI F et al. Early interim 2-(18F)fluoro-2-deoxy-D- glucose positron emission tomography is prognostically superior to International Prognostic Score in advanced-stage Hodgkins lymphoma: A report from a joint Italian- Danish study. J ClinOncol.2007; 25: 3746-3752. http://dx.doi.org/10.1200/ LCO.2007.11.6525

[4] KOSTAKOGLU L, GALLAMINI A. Interim 18F-FDG PET in Hodgkin Lymphoma: Would PET-Adapted Clinical Trials Lead to a Paradigm Shift? J NuclMed . 2013; 54 (7): 1082-1093. http://dx.doi.org/10.2967/jnumed.113.120451 
[5] MEIGNAN M, GALLAMINI A, MEIGNAN M, GALLAMINI A, HAIOUN C. Report on the First International Workshop on Interim-PET-Scan in Lymphoma. Leuk Lymph.2009; 50(8): 1257-1260. http://dx.doi.org/10.1080/10428190903040048

6] HUTCHINGS M, MIKHAEEL NG, FIELDS PA, NUNAN T, TIMOTHY AR. Prognostic value of interim FDG-PET after two or three cycles of chemotherapy in Hodgkin lymphoma. Ann Oncol. 2005; 16: 1160-1168. http://dx.doi.org/10.1093/ annonc/mdi200

[7] ZINZANI PL, RIGACCI L, STEFONI V, BORCCOLI A, PUCCINI B et al. Early interim 18F-FDG PET in Hodgkins lymphoma: evaluation on 304 patients. Eur J Nucl Med Mol Imaging 2012; 39: 4-12. http://dx.doi.org/10.1007/s00259-011-1916-8

[8] STRAUS DJ, JOHNSON JL, LACASCE AS, BARTLETT NL, KOSTAKOGLU L et al. Doxorubicin, vinblastine, and gemcitabine (CALGB 50203) for stage I/II nonbulky Hodgkin lymphoma: pretreatment prognostic factors and interim PET. Blood; 2011; 117: 5314-5320. http://dx.doi.org/10.1182/ blood-2010-10-314260

[9] CERCI JJ, PRACCHIA LF, LINARDI CC, PITELLA FA, DELBEKE $D$ et al. 18F-FDG PET after 2 cycles of ABVD predicts event-free survival in early and advanced Hodgkin lymphoma. J Nucl Med 2010; 51: 1337-1343. http://dx.doi. org/10.2967/jnumed.109.073197

[10] BARNES JA, LACASCE AS, ZUKOTYNSKI K, ISRAEL D, FENG $\mathrm{Y}$ et al. End-of-treatment but not interim PET scan predicts outcome in nonbulky limited-stage Hodgkin's lymphoma. Ann Oncol; 2011; 22: 910-915. http://dx.doi. org/10.1093/annonc/mdq549

[11] FILIPPI AR, BOTTICELLA A, BELLO M, BOTTO B, CASTIGLIONE A et al. Interim positron emission tomography and clinical outcome in patients with early stage Hodgkin lymphoma treated with combined modality therapy. Leuk Lymphoma 2013; 54: 1183-1187. http://dx.doi.org/10.310 9/10428194.2012.735667

[12] HUTCHINGS M. FDG-PET Response-adapted Therapy Is 18F-Fluorodeoxyglucose Positron Emission Tomography a Safe Predictor for a Change of Therapy? HematolOncolClin N Am 2014; 28: 87-103.

[13] DANN EJ, BAR-SHALOM R, TAMIR A, EPELBAUM L, AVIVI I ET AL. A functional dynamic scoring model to elucidate the significance of post-induction interim fluorine-18-fluorodeoxyglucose positron emission tomography findings in patients with Hodgkin's lymphoma. Haematol.2010; 95: 1198-206. http://dx.doi.org/10.3324/ haematol.2009.016105

[14] BIGGI A, GALLAMINI A, CHAUVIE S, HUTCHINGS M, KOSTAKOGLU L ET AL. International validation study for interim PET in ABVD-treated, advanced-stage hodgkin lymphoma: interpretation criteria and concordance rate among reviewers. J Nucl Med. 2013; 54(5): 683-90. http://dx.doi. org/10.2967/jnumed.112.110890 